\title{
Cardiac regeneration in the axolotl is unaffected by alterations in leukocyte numbers induced by lipopolysaccharide and prednisolone
}

\author{
Kathrine Pedersen ${ }^{\dagger}$, Rikke Kongsgaard Rasmussen ${ }^{\dagger}$, Anita Dittrich and Henrik Lauridsen $^{*}$ (]
}

\begin{abstract}
Objective: Cardiac regeneration in the axolotl has been found to rely on the innate immune system, and especially macrophages have been demonstrated to play a vital role in regulating the regenerative process. In this study we wanted to induce a pro- and anti-inflammatory milieu in the axolotl during heart regeneration to test the resilience of the regenerative response.

Results: This was induced via repeated intrapericardial injections of lipopolysaccharide or prednisolone during a 40-day regeneration period in order to challenge the presumably fine-tuned inflammatory response that normally facilitates regeneration. We observed a local and systemic leucocyte response to pro- and anti-inflammatory stimulation, but we found cardiac regeneration to be structurally and functionally unaffected.
\end{abstract}

Keywords: Heart regeneration, Axolotl, Inflammation, Immune system, Lipopolysaccharide, Prednisolone

\section{Introduction}

The axolotl is a popular model organism in the field of regenerative medicine, due to its ability to regenerate for instance functional heart muscle after injury [1]. In adult mammals, cardiac injury leads to fibrotic scarring. The response to injury in fetal mammals is dominated by anti-inflammatory macrophages promoting repair, while pro-inflammatory macrophages are more abundant in the adult, causing fibrosis [2]. Even though possible macrophage-polarizations are not well described in the axolotl, macrophages have been shown to be critical for regeneration of cardiac muscle [3] and amputated limbs [4].

\footnotetext{
*Correspondence: henrik@clin.au.dk

${ }^{\dagger}$ Kathrine Pedersen and RikkeKongsgaard Rasmussen contributed equally to this work

Department of Clinical Medicine (Comparative Medicine Lab), Aarhus University, 8200 Aarhus N, Denmark
}

We aimed to test the resilience of the pro-regenerative inflammatory response in the axolotl upon challenge with lipopolysaccharide (LPS), prednisolone or saline control. LPS is strongly pro-inflammatory and can induce proinflammatory macrophage polarization [5], while prednisolone can inhibit inflammatory macrophage activity and promote anti-inflammatory-polarization in vitro $[6,7]$.

\section{Main text \\ Methods}

\section{Animal experiments}

Axolotls (weight $\pm \mathrm{SD}=18.1 \pm 3.3 \quad$ g; total length $\pm \mathrm{SD}=13.3 \pm 0.9 \mathrm{~cm}$ ) were housed individually at $20{ }^{\circ} \mathrm{C}$ on a 12 -h light/dark cycle. Animals from a commercial breeder (Exoterra GmbH, Germany) were acclimatized 14 days prior to experimental procedures. All procedures were conducted in dedicated laboratory spaces. General anesthesia was obtained by submersion in benzocaine solution (200 $\left.\mathrm{mg} \mathrm{l}^{-1}, 30 \mathrm{~min}\right)$ prior to echocardiography, 
intrapericardial injections and cryoinfarction surgery. For cryoinfarction surgery, the ventricle was exposed, and a copper probe cooled in liquid nitrogen was applied to the ventroapical face of the heart for $10 \mathrm{~s}$. Incision was closed with individual stiches. Injections were performed by inserting a syringe carrying a 30 -gauge needle into the pericardial space. Axolotls were injected every five days starting 5 days prior to cryoinfarction surgery with $20 \mu \mathrm{l}$ of either sterile amphibian Ringer's solution $\left(6.6 \mathrm{~g} \mathrm{l}^{-1} \mathrm{NaCl}\right.$, $0.15 \mathrm{~g} \mathrm{l}^{-1} \mathrm{KCl}, 0.15 \mathrm{~g} \mathrm{l}^{-1} \mathrm{CaCl}_{2}, 0.2 \mathrm{~g} \mathrm{l}^{-1} \mathrm{NaHCO}_{3}$ ) for saline control, $10 \mu \mathrm{g}$ of LPS (Sigma Aldrich, L5886) or $20 \mu \mathrm{g}$ of prednisolone (Sigma Aldrich, P6004), both dissolved in Ringer's solution. Initial sample size was 12 animals per group. However, due to insufficient knowledge about effect size, power calculations could not be conducted. Forty days post cryoinfarction, animals were euthanized by overdosing animals in benzocaine and harvesting hearts blood and pericardial aspirate.

\section{Echocardiography}

Starting 5 days prior to injury, animals were imaged with echocardiography every five days up to day 40 (both preand post-injury on day 0 ). Imaging started exactly $30 \mathrm{~min}$ after initializing anesthesia and the imaging protocol was identical between animals, due to the cardio-stimulatory effect of benzocaine over time [8]. Animals were imaged in a supine position submerged in water using the Visualsonics Vevo 2100 system and a MS700 (48-50 MHz) transducer as previously described [9]. B-mode recordings (1000 frames, frame rate range of $45-65 \mathrm{f} \mathrm{s}^{-1}$ ) of the ventricle in long-axis view, color-Doppler recordings of the ventricle and pulsed wave Doppler recordings in the outflow tract were obtained for heart rate measurements. Three cardiac cycles were analyzed for each data point using Visualsonics Vevo LAB ultrasound analysis software to measure mid ventricular cross-sectional area at end diastole, end systole as well as mid infarction crosssectional area and heart rate (HR) to calculate infarction fraction (IF), healed fraction (HF), stroke volume (SV) and cardiac output (CO).

HF of the infarction was calculated at each time point (t) with IF at day 5 as reference:

$$
H F(t)=\frac{I F(\operatorname{day} 5)-I F(t)}{I F(\text { day } 5)} \times 100 \%
$$

SV was calculated from end diastolic area (EDA) and end systolic area (ESA) under the assumption of a spherical ventricle:

$$
S V=\frac{4}{3} \times \pi \times \sqrt{\frac{E D A}{\pi}}^{3}-\frac{4}{3} \times \pi \times \sqrt{\frac{E S A}{\pi}}^{3}
$$

CO was calculated from HR, SV and weight:

$$
C O=\frac{H R \times S V}{w e i g h t}
$$

\section{Collection of ventricle, blood and aspirate}

At day 40 animals were anesthetized, and an incision was made into the pericardium to collect pericardial fluid. Pericardial aspirate smears were prepared immediately and fixated in ice cold methanol for $2 \mathrm{~min}$. Axolotls were then injected with $50 \mu \mathrm{l}$ of 2,500 $\mathrm{IU} \mathrm{ml} \mathrm{m}^{-1}$ heparin and the outflow tract was severed to collect arterial blood. Blood smears were prepared in the same way as aspirate smears. The ventricle was harvested, gently rinsed with amphibian Ringer's solution and transferred to $4 \%$ neutral buffered formalin.

\section{Histology}

Fixated aspirate and blood smears were stained in modified Wright-Giemsa staining solution (Sigma Aldrich, cat. nr: WG128) for $45 \mathrm{~s}$, rinsed in deionized water and mounted with DPX mountant (Sigma Aldrich, cat. nr: 06522). Formalin fixed ventricles were embedded in paraffin and sectioned at $10 \mu \mathrm{m}$ slice thickness across the entire ventricle, with every fifth section collected and stained using a Masson's-trichrome kit (Polysciences Inc., cat. nr: 25088) according to the manufacturers protocol. Unbiased stereology was performed as described by Mühlfeld et al. [10] to measure ventricle and infarction volume using a minimum of 12 evenly spaced sections across each heart and counting $>100$ point grid intersections with myocardial tissue.

\section{Statistical analysis}

Relevant t-tests (paired/unpaired) were used to test for statistically significant differences between two groups. Relevant types of ANOVAs (repeated measure, one-way/ two-way) were used for omnibus testing of statistically significant difference between more than two groups and evaluate differences between treatment groups and between time points. For post hoc tests of difference between groups, Bonferroni corrected $\mathrm{t}$-tests were used. Significance level $(\alpha)=0.05$ unless specified as Bonferroni correction. Unless otherwise specified, reported measurements throughout the text and figures are arithmetic mean $\pm 95 \%$ confidence interval.

\section{Results and discussion}

Continuous injections with either LPS, prednisolone or saline (Fig. 1a), resulted in significant differences between leukocyte counts between treatment groups at day 40 (two-way ANOVA with regression, $\mathrm{p}=0.011$ ). Post hoc $\mathrm{t}$-tests showed increased numbers of several types of leukocytes in arterial blood in LPS treated axolotls 

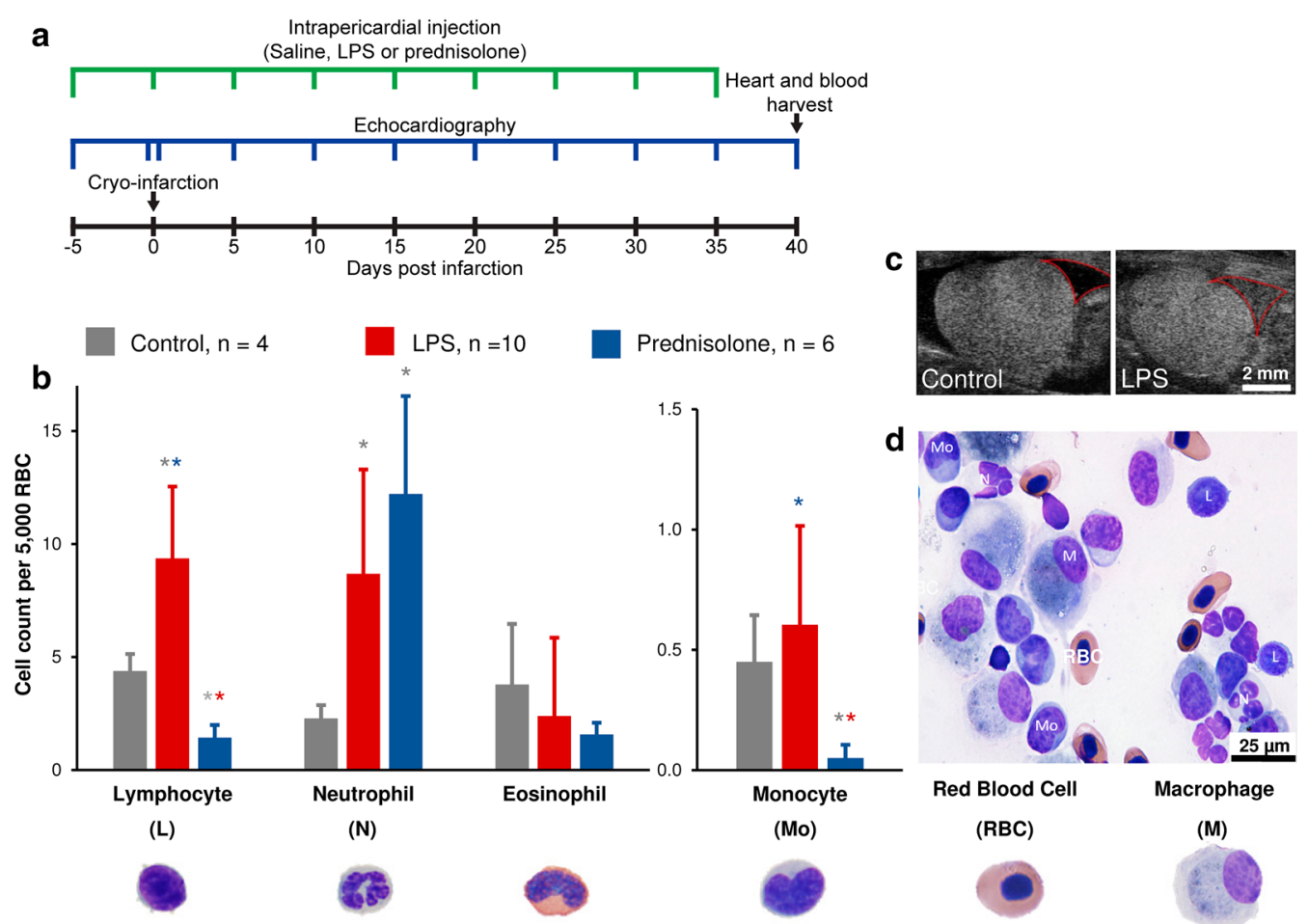

Fig. 1 Systemic and local inflammatory response to pro- and anti-inflammatory stimulation. a Graphical overview of experiment. b Leukocyte numbers expressed as cell count per 5,000 red blood cell (RBC) on Wright-Giemsa stained blood smears. Significant differences shown by asterisk (grey indicating significant difference to control, red to LPS and blue to prednisolone). Representative images of axolotl leukocytes are displayed below each cell category. Error bars represent 95\% confidence interval. Two-tailed unpaired students t-test with $a=0.05$ was used to determine statistical significance. c Representative echocardiography images of control (left) and LPS (right) treated animals with clear pericardial fluid in control and pericardial fluid with nucleated cell infiltrate in LPS (area marked with red line). $\mathbf{d}$ Representative image of Wright-Giemsa stained smear of pericardial aspirate from LPS treated animal

compared to both control and prednisolone groups (Fig. 1b). The number of lymphocytes and neutrophils were significantly increased in LPS treated compared to control animals (unpaired t-test, lymphocytes: $\mathrm{p}=0.014$; neutrophils: $\mathrm{p}=0.025)$. Prednisolone treated animals in turn showed significant decrease in numbers of lymphocytes and monocytes compared to control (unpaired t-test, lymphocytes: $\mathrm{p}<0.01$; monocytes: $\mathrm{p}<0.01$ ) but an increase in neutrophil number (unpaired t-test, $\mathrm{p}<0.01$ ), reflecting a known effect of prednisolone [11], which does not necessarily indicate increased neutrophil activity. Lymphocyte and monocyte numbers were also significantly higher in LPS compared to prednisolone animals (unpaired t-test, lymphocytes: $\mathrm{p}<0.01$; monocytes: $\mathrm{p}<0.01)$.

A local inflammatory response in the pericardial space was observed in LPS animals on echocardiography, which showed gradual accumulation of nucleated cells (Fig. 1c). Pericardial fluid aspirated after 40 days of regeneration contained large numbers of leukocytes in the LPS group compared to control and prednisolone groups (Fig. 1d). The lowest level of infiltrate was seen in the prednisolone group. Pericardial aspirate from the LPS group was dominated primarily by macrophages and secondly monocytes, lymphocytes and neutrophils (Fig. 1d).

The level of regeneration and cardiac function was assayed longitudinally by echocardiography (Figs. 1a, $2 a-d)$. Structural regeneration was evaluated by the size of the infarction zone measured as ventricle volume not contracting. Immediately after cryoinjury, mean infarction fraction constituted $8.6 \pm 1.4 \%$ of ventricle volume across all groups $(n=24)$. Infarction fraction significantly increased (paired t-test, $\mathrm{n}=24, \mathrm{p}<0.01$ ) to constitute $18.3 \pm 4.3 \%$ of ventricle volume five days after cryoinjury where the injury zone was fully developed. No significant difference was observed in fold change of infarction fraction between groups (One-way ANOVA, $\mathrm{p}=0.74)$. To assess anatomical regeneration, maximum infarction size at five days after injury was used as reference to measure subsequent healed fraction until 40 days after injury (Fig. 2a). The infarction zone was gradually regenerated (Fig. 2a) (Two-way ANOVA with repeated measures, $\mathrm{p}<0.01$ with Bonferroni corrected $(\alpha / 7=0.0071)$ post hoc t-tests showing 


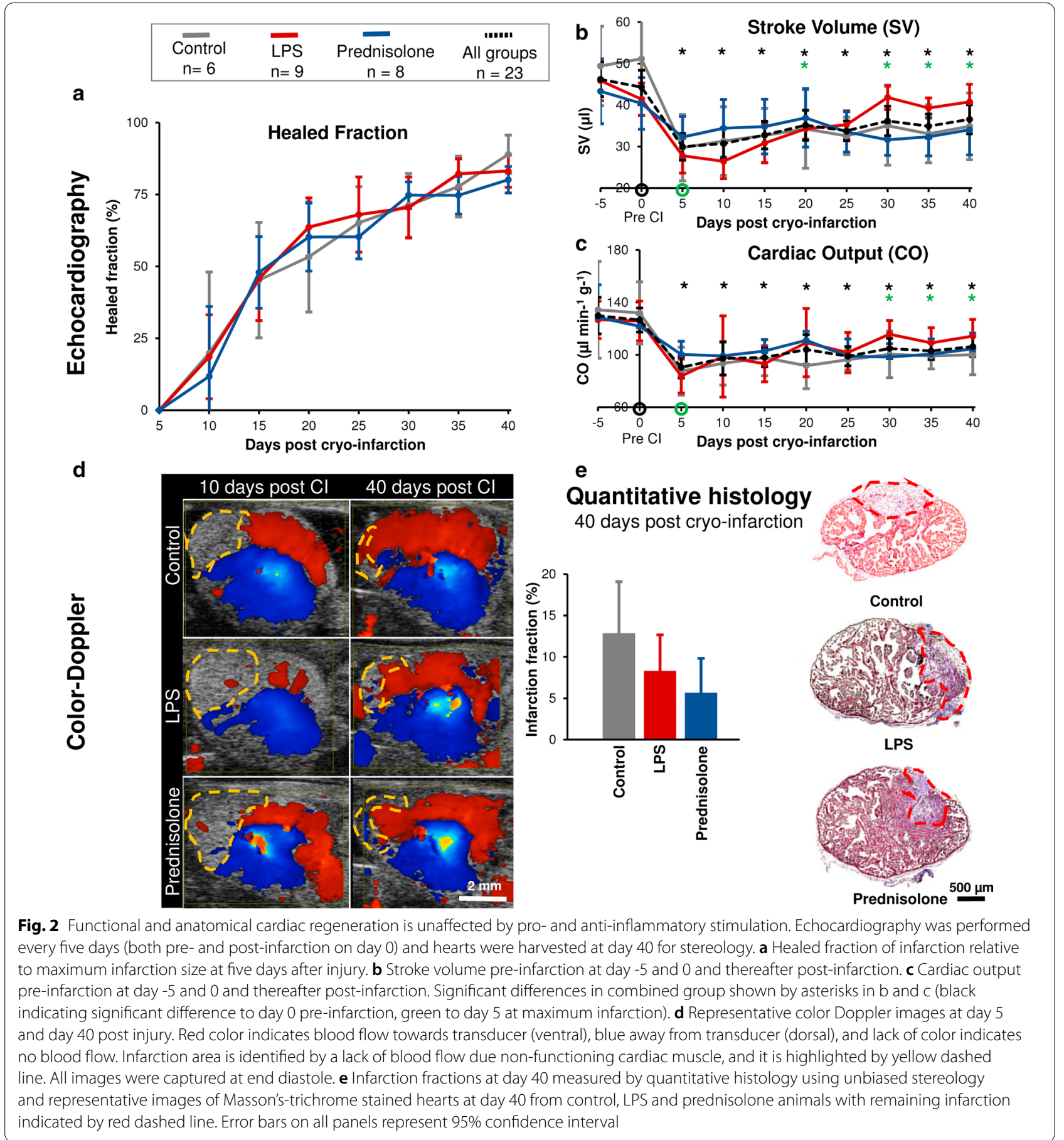

significant increased healed fraction in all groups from 15 days until 40 days post injury). No significant difference was observed in healed fraction between groups at any time point (Two-way ANOVA with repeated measures, $\mathrm{p}=0.96) .40$ days after injury, the healed fraction was $83.8 \pm 3.4 \%$ across groups $(\mathrm{n}=23)$.
Quantitative histology using unbiased stereology, measuring infarction fraction on Masson's trichromestained sections across entire hearts harvested 40 days post injury showed no significant difference in remaining infarction fraction (One-way ANOVA, p=0.17) (Fig. 2e) or ventricular myocardial volume between groups 
(One-way ANOVA, $\mathrm{p}=0.58$ ). Across groups ventricular myocardial volume was $2.47 \pm 0.33 \mu \mathrm{l} 40$ days after injury and the remaining infarction fraction was $8.45 \pm 2.90 \%$.

Stroke volume and cardiac output was used to evaluate functional recovery after cryoinjury. Cryoinjury significantly affected cardiac function for both stroke volume (Two-way ANOVA with repeated measures, $\mathrm{p}<0.01$ ) and cardiac output (Two-way ANOVA with repeated measures, $\mathrm{p}<0.01)$. However, functional regeneration progressed similarly with no significant difference between groups for both stroke volume (Two-way ANOVA with repeated measures, $\mathrm{p}=0.98$ ) and cardiac output (Two-way ANOVA with repeated measures, $\mathrm{p}=0.54$ ). Five days after injury stroke volume and cardiac output across all groups was significantly reduced to $69.0 \pm 6.4 \%$ and $72.7 \pm 6.6 \%$, respectively, compared to immediately before injury (Paired t-tests, $n=24$; Stroke volume: $\mathrm{p}<0.001$; Cardiac output: $\mathrm{p}<0.01$ ). Thirty days after cryoinjury stroke volume and cardiac output were significantly increased compared to minimum function at 5 days after injury (Bonferroni corrected $(\alpha / 7=0.0071)$ post hoc t-tests) (Fig. 2b, c). This demonstrated functional recovery, although not to the initial level since stroke volume and cardiac output were significantly lower at 40 days after injury compared to pre-injury; $82.5 \pm 10.0 \%$ and $87.4 \pm 6.8 \%$ respectively (Paired t-tests, $\mathrm{n}=23$; Stroke volume: $\mathrm{p}<0.01$; Cardiac output: $\mathrm{p}<0.01$ ) (Fig. 2b, c). Imaging the heart using color Doppler imaging, qualitatively showed compromised blood flow in the infarcted area in early time points after injury, which was close to normal after 40 days of regeneration with no difference between groups (Fig. 2d).

Taken together, our results strongly suggests that although there are alterations in local and systemic leukocyte numbers in response to LPS and prednisolone, it does not affect cardiac function nor the rate or fidelity of cardiac regeneration following injury in the axolotl compared to control animals. As previously demonstrated, LPS stimulation does alter cytokine profiles in the axolotl [11], thus supporting that the altered leukocyte counts found here in response to LPS injections represents an altered inflammatory cardiac environment.

With a previous demonstration of the importance of macrophage function in axolotl cardiac regeneration [3], it is somewhat surprising that structural and functional cardiac regeneration is unaffected by significant alterations in leukocyte numbers. Polarization of macrophages in vivo is believed to be on a spectrum, rather than the M1/M2 phenotypes described in vitro [12], however, it remains central to the understanding of macrophage phenotypes.

One hypothesis of how the immune response is more favorable towards regeneration in fetal and neonatal mammals as well as regenerative model organisms like the zebrafish and the axolotl is that the more naïve immunological state in these organisms falls short of mounting a substantial pro-inflammatory response to injury with the benefit of reduced fibrosis and increased ability for regeneration as reviewed by Sattler and Rosenthal [13]. This does, however, seem somewhat counterintuitive to the massive increase in leukocyte numbers observed in response to LPS in the current study, with cardiac regeneration proceeding unaffected. It may also be indicative of critical differences in the axolotl versus mammalian immune function with respect to regeneration. A comparative study of the cardiac regeneration competent zebrafish and the cardiac regeneration incompetent medaka, brings the idea of a low-level immune response being favorable for regeneration further into questioning. It was shown that the regenerating zebrafish compared to the medaka, in fact mount a strong inflammatory response more quickly with an increased level of macrophage infiltration, which in turn cleared more efficiently [14].

Deducing the exact phenotype of macrophages involved in cardiac regeneration in the axolotl will be highly relevant to further the understanding of the regenerative process and the knowledge may one day be translated into human therapies. The tolerance of the macrophage dependent regenerative response to LPS and prednisolone indicate that the reparative phenotype is not subject to polarization towards a non-reparative proinflammatory -type via LPS/toll-like receptor signaling or anti-inflammatory inactivation by corticosteroid/prednisolone signaling, although these clearly have an effect on leukocyte numbers both locally and systemically.

\section{Limitations}

A valuable sophistication of the experiment would be repeated blood sampling during the regenerative process in the axolotl groups to allow for leukocyte counting and cytokine profiling throughout the regenerative process. This would however require larger animals to be ethically acceptable.

\section{Abbreviations}

LPS: Lipopolysaccharide; SD: Standard deviation; B-mode: Bright mode; HR: Heart rate; IF: Infarction fraction; HF: Healed fraction; SV: Stroke volume; CO: Cardiac output; t:Time; EDA: End diastolic area; ESA: End systolic area; BM: Body mass.

\section{Acknowledgements}

The authors would like to thank the Department of Forensic Medicine (Aarhus University) and the Institute of Pathology (Aarhus University Hospital) for assistance and use of facilities for histology.

\section{Authors' contributions}

$\mathrm{HL}$ and $\mathrm{AD}$ conceived, designed and supervised the experiments. AD performed axolotl surgery while the remaining experimental work including 
echocardiography, sample collection and histology as well as data analysis was performed by KP and RKR. AD drafted the manuscript and KP, RKR and HL made revisions. All authors read and approved the final manuscript.

\section{Funding}

The A.P. Møller Foundation for the Advancement of Medical Science, The Velux Foundations, and The Lundbeck Foundation provided funding for the study.

\section{Availability of data and materials}

The datasets used and/or analyzed during the current study are available from the corresponding author $(\mathrm{HL})$ on reasonable request.

\section{Declarations}

\section{Ethics approval and consent to participate}

The animal experiments and housing facilities are in accordance with Danish Legislation, and approved by the Danish National Animal Experiments expectorate (Dyreforsøgstilsynet) under protocol 2015-15-020100615.

\section{Consent for publication}

Not applicable.

\section{Competing interests}

The authors declare no competing interests.

Received: 18 December 2020 Accepted: 15 April 2021

Published online: 28 April 2021

\section{References}

1. Cano-Martínez A, et al. Functional and structural regeneration in the axolotl heart (Ambystoma mexicanum) after partial ventricular amputation. Archivos de Cardiologia de Mexico. 2010;80:79-86.

2. Aurora $A B$, et al. Macrophages are required for neonatal heart regeneration. J Clin Invest. 2014;124:1382-92.

3. Godwin JW, Debuque R, Salimova E, Rosenthal NA. Heart regeneration in the salamander relies on macrophage-mediated control of fibroblast activation and the extracellular landscape. NPJ Regen Med. 2017;2:22.
4. Godwin JW, Pinto AR, Rosenthal NA. Macrophages are required for adult salamander limb regeneration. Proc Natl Acad Sci U S A. 2013:110:9415-20.

5. Wu TT, Chen TL, Chen RM. Lipopolysaccharide triggers macrophage activation of inflammatory cytokine expression, chemotaxis, phagocytosis, and oxidative ability via a toll-like receptor 4-dependent pathway: Validated by RNA interference. Toxicol Lett. 2009:191:195-202.

6. Lim HY, Müller N, Herold MJ, Van Den Brandt J, Reichardt HM. Glucocorticoids exert opposing effects on macrophage function dependent on their concentration. Immunology. 2007;122:47-53.

7. Paulus $\mathrm{P}$, et al. Prednisolone as preservation additive prevents from ischemia reperfusion injury in a rat model of orthotopic lung transplantation. PLoS ONE. 2013;8:e73298.

8. Thygesen MM, Rasmussen MM, Madsen JG, Pedersen M, Lauridsen H. Propofol (2,6-diisopropylphenol) is an applicable immersion anesthetic in the axolotl with potential uses in hemodynamic and neurophysiological experiments. Regeneration. 2017:4:124-31.

9. Dittrich A, Thygesen MM, Lauridsen H. 2D and 3D echocardiography in the axolotl (Ambystoma mexicanum). J Vis Exp. 2018;141:e57089.

10. Mühlfeld C, Nyengaard JR, Mayhew TM. A review of state-of-the-art stereology for better quantitative 3D morphology in cardiac research. Cardiovasc Pathol. 2010;19:65-82.

11. Ronchetti S, Ricci E, Migliorati G, Gentili M, Riccardi C. How glucocorticoids affect the neutrophil life. Int J Mol Sci. 2018;19:e4090.

12. Mosser DM, Edwards JP. Exploring the full spectrum of macrophage activation. Nat Rev Immunol. 2008;8:958-69.

13. Sattler S, Rosenthal $\mathrm{N}$. The neonate versus adult mammalian immune system in cardiac repair and regeneration. Biochim Biophys Acta. 2016;1863:1813-21.

14. Lai SL, et al. Reciprocal analyses in zebrafish and medaka reveal that harnessing the immune response promotes cardiac regeneration. Elife. 2017;6:e25605.

\section{Publisher's Note}

Springer Nature remains neutral with regard to jurisdictional claims in published maps and institutional affiliations.
Ready to submit your research? Choose BMC and benefit from:

- fast, convenient online submission

- thorough peer review by experienced researchers in your field

- rapid publication on acceptance

- support for research data, including large and complex data types

- gold Open Access which fosters wider collaboration and increased citations

- maximum visibility for your research: over $100 \mathrm{M}$ website views per year

At BMC, research is always in progress.

Learn more biomedcentral.com/submissions 